\section{ORIGINAL RESEARCH}

\author{
A.A.K. Abdel Razek \\ A.Y. Kandeel \\ N. Soliman \\ H.M. El-shenshawy \\ Y. Kamel \\ N. Nada \\ A. Denewar
}

\title{
Role of Diffusion-Weighted Echo-Planar MR \\ Imaging in Differentiation of Residual or Recurrent Head and Neck Tumors and Posttreatment Changes
}

\begin{abstract}
BACKGROUND AND PURPOSE: The purpose of this work was to evaluate whether diffusion-weighted MR imaging can be used in differentiating residual or recurrent head and neck tumors from postoperative or postradiation changes
\end{abstract}

MATERIALS AND METHODS: This study included 32 patients clinically suspected for recurrent head and neck tumor after surgery $(n=3)$, radiation therapy $(n=13)$, or both $(n=16)$. Diffusion-weighted MR imaging was done by using a single-shot spin-echo echo-planar sequence. The apparent diffusion coefficient $(A D C)$ value of the suspected lesion was calculated and correlated with pathologic results.

RESULTS: Adequate diffusion-weighted MR images and ADC maps were obtained in 30 patients (93.8\%). The mean ADC value of residual or recurrent lesions $\left(1.17 \pm 0.33 \times 10^{-3} \mathrm{~mm}^{2} / \mathrm{s}\right)$ was less than that of posttherapeutic changes $\left(2.07 \pm 0.25 \times 10^{-3} \mathrm{~mm}^{2} / \mathrm{s}\right)$, and the difference was statistically significant $(P<.001)$. When an ADC value of $1.30 \times 10^{-3} \mathrm{~mm}^{2} / \mathrm{s}$ was used as a threshold value for differentiation, the best results were obtained with an accuracy of $87 \%$, sensitivity of $84 \%$, specificity of $90 \%$, positive predictive value of $94 \%$, and negative predictive value of $76 \%$.

CONCLUSIONS: Diffusion-weighted MR imaging with ADC measurement has promising results for differentiating residual or recurrent head and neck tumors from postoperative or postradiation changes.
$\mathbf{S}_{n}$ urgery, radiation therapy, and/or chemotherapy are the mainstays of treatment of head and neck cancer. ${ }^{1}$ The examination of patients with head and neck cancer after treatment poses special problems. After surgery, normal anatomic structures can be extensively distorted. The use of radiation therapy renders physical examination, CT, and MR imaging unreliable because of the edema and fibrosis that are often present after treatment. Biopsy is often necessary, but the results of histopathologic specimens can be inaccurate because of sampling errors. ${ }^{2-5}$

Diffusion-weighted MR imaging is based on the diffusion motion of water protons in the tissues. When measuring molecular motion with diffusion-weighted MR imaging, only the apparent diffusion coefficient (ADC) can be calculated. ${ }^{6,7}$ ADC values are expected to vary according to the microstructures or pathophysiologic states of the tissues, and they can be calculated by measuring signal intensities in a series of diffusion-weighted MR images by using different b-values. ${ }^{7,8}$

Diffusion-weighted MR imaging has been successfully used in the diagnosis of many disorders of the central nervous system, ${ }^{6}$ liver, ${ }^{9}$ and bone marrow. ${ }^{10}$ Promising results have also been achieved for differentiation between residual or recurrent tumors and postradiation or postsurgical changes in soft tissues ${ }^{11}$ and the brain. ${ }^{12}$ Recent advances in echo-planar technique have permitted the clinical use of diffusionweighted MR imaging in the head and neck. ${ }^{7,13,14}$ The aim of

Received June 27, 2006; accepted after revision October 10.

From the Departments of Diagnostic Radiology (A.A.K.A.R., A.Y.K., N.S.), Clinical Oncology and Nuclear Medicine (H.M.E.), ENT and Head and Neck Surgery (Y.K.), Oncology Surgery (A.D.), and Pathology (N.N.), Faculty of Medicine, Mansoura University, Mansoura, Egypt. Address correspondence to Ahmed Abdel Khalek Abdel Razek, Diagnostic Radiology Department, Mansoura Faculty of Medicine, 62 El Nokrasi St, Mansoura, Egypt; e-mail: arazek@mans.eun.eg

DOI 10.3174/ajnr.A0491 this study was to evaluate the role of diffusion-weighted MR imaging in differentiating residual or recurrent head and neck tumor from postoperative or postradiation changes.

\section{Materials and Methods}

This prospective study was performed on 32 consecutive patients ( 24 men and 8 women aged $45-71$ years; mean age, 58 years) after surgical resection and/or radiation therapy who were suspected to have residual or recurrent tumor of the head and neck. They were prospectively evaluated with MR imaging including diffusion-weighted MR imaging. Patients were referred from the follow-up clinic of the radiation therapy and oncology department at our hospital. Patients were evaluated because of recurrence of symptoms $(n=20)$ or abnormal physical examination findings during routine follow-up $(n=12)$. The approval of the institutional review board was obtained, and a written consent was obtained from all of the patients.

Pathology of primary tumors included the following: squamous cell carcinoma $(n=20)$, mucoepidermoid carcinoma $(n=2)$, adenoid cystic carcinoma $(n=2)$, adenocarcinoma $(n=3)$, oncocytic adenocarcinoma $(n=2)$, and sarcoma $(n=3)$. These tumors were located as follows: nasopharynx $(n=8)$, oropharynx $(n=4)$, larynx $(n=4)$, paranasal sinuses and nasal cavity $(n=8)$, salivary glands $(n=6)$, skull base $(n=1)$, and cheek $(n=1)$. These lesions were treated with surgery alone in 3 patients, radiation therapy alone in 13 patients, or both surgery and radiation therapy in the remaining 16 patients. The total radiation dose ranged from 60 to 70 Gy given by conventional fractionation (1.8-2.0 Gy per fraction), with 5 fractions per week over 6-7.5 weeks. The filed arrangement varied according to the site of the primary lesions. The size of the irradiated fields was chosen according to the extension of the tumor, stage, and probability of lymphatic spread. The lower neck and supraclavicular fossa were usually treated with single anterior filed to $50 \mathrm{~Gy}$ per given dose, with 2 Gy daily fractions, given over 5 weeks. Megavoltage irradiation with 


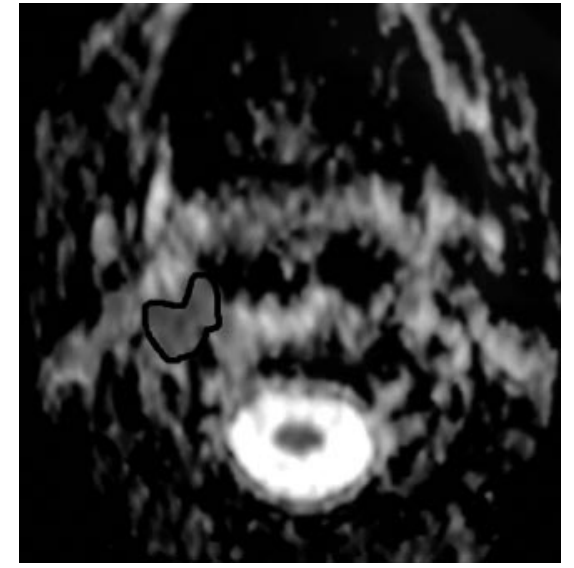

Fig 1. $A D C$ map demonstrating the location of $\mathrm{ROI}$ in a patient with a recurrent nasopharyngeal mass.

6-10 megavoltage photons was used. MR examinations were done within a period of $4-14$ months after treatment.

All of the MR studies were performed with a 1.5 T MR machine (Symphony; Siemens Medical Systems, Erlangen, Germany) by using a head and/or neck circular polarization surface coil. All of the patients underwent T1-weighted images (T1WIs; TR/TE of 800/15 ms) and T2-weighted fast spin-echo images (TR/TE of $4500 / 80 \mathrm{~ms}$ ) with a section thickness of $5 \mathrm{~mm}$, an intersection gap of 1-2 mm, an FOV of $23 \times 23 \mathrm{~cm}$, and an acquisition matrix of $224 \times 256$.

Diffusion-weighted MR images were obtained by using a multisection spin-echo single-shot echo-planar imaging (EPI) sequence. An average of $\sim 15$ sections was obtained in the axial plane covering the area of interest. Imaging parameters were as follows: TR/TE of $10,000 / 108 \mathrm{~ms}$, FOV of $23 \times 23 \mathrm{~cm}$, an acquisition matrix of $128 \times$ 128 , and section thickness of $5 \mathrm{~mm}$ with an intersection gap of $1-2$ $\mathrm{mm}$. Diffusion-probing gradients were applied in the 3 orthogonal directions (X, Y, and Z) with the same strength. Diffusion-weighted MR images were acquired with diffusion-weighted factor, factor $b$, of 0 and $1000 \mathrm{~s} / \mathrm{mm}^{2}$, and ADC maps were generated for all of the images. Scanning time was $<1$ minute. Finally, postcontrast T1WIs (TR/TE of 800/15 ms) were obtained after an intravenous bolus injection of $0.2 \mathrm{~mL} / \mathrm{kg}$ of body weight of gadopentetate dimeglumine (Magnevist; Schering, Berlin, Germany) in all of the patients.

The quality of the diffusion-weighted MR images was evaluated by the 3 radiologists sharing in the study, and it was determined by consensus whether they were acceptable for further analysis. Special attention was paid to image distortion by susceptibility artifacts. A qualitative and quantitative analysis of the diffusion-weighted MR images was made. Two radiologists were asked, independently, to subjectively assess the signal intensity of the areas of suspicion on the diffusion-weighted MR images and the ADC maps and to grade it as low, high, or mixed signal intensity. The third radiologist measured the $\mathrm{ADC}$ of the suspicious areas by using an electronic cursor to define region of interest (ROI).

An ROI was placed around the margin of the suspicious area, and the ADC value was measured (Fig 1). When heterogeneity in signal intensity was observed, macrocystic regions were excluded, and multiple small ROIs were placed within the cross-section of the suspicious area with calculation of the mean ADC value. Similarly, the routine MR images (T1WI, T2WI, and postcontrast T1WI) were evaluated by the 3 radiologists independently for evidence of residual or recurrent tumor versus posttreatment changes. Signs suggestive of recurrence

\begin{tabular}{lc}
\hline $\begin{array}{l}\text { Table 1: Location and method of treatment in } \mathbf{3 0} \text { examinations that } \\
\text { qualified for our study }\end{array}$ \\
\hline $\begin{array}{l}\text { No. of } \\
\text { Patients }\end{array}$ \\
Patient Characteristic & \\
\hline Location & 8 \\
Nasopharynx & 4 \\
Oropharynx & 3 \\
Larynx & 7 \\
Paranasal sinuses and nasal cavity & 6 \\
Salivary glands & 1 \\
Skull base & 1 \\
Cheek & \\
Method of treatment & 3 \\
Surgery & 12 \\
Radiotherapy & 15 \\
Surgery and radiotherapy
\end{tabular}

on routine MR imaging were areas of $\mathrm{T} 2$ prolongation, variable mass effect, and enhancement after the administration of gadolinium-diethylene-triaminepentaacetic acid. None of the 3 radiologists was aware of the location of the primary tumor, its histologic type, clinical stage, or the results of any recent biopsy, if taken, at the time of analysis of the MR studies. The analysis of diffusion-weighted images was done at the same time with routine MR.

The final diagnosis in our study was confirmed by biopsy in all of the patients. Biopsy was done for all of the patients after MR imaging. The selection of the site of biopsy was guided by the findings on conventional and diffusion-weighted MR imaging. The surgeons selected the biopsy site after discussing the MR findings with the radiologists. Biopsy was done by surgical biopsy in 14 cases, core biopsy in 11 patients, and fine needle aspiration biopsy in 7 patients. When there was suspicion of more than one pathologic condition, multiple biopsies were taken from the suspicious sites. The time delay between the biopsy and diffusion MR study varied between 7 and 13 days. The specimens were interpreted by 1 pathologist experienced in head and neck.

The mean ADC values of residual or recurrent tumors and posttreatment changes were compared by using the Mann-Whitney test. SPSS statistical software package, version 9 (SPSS, Chicago, Ill), was used for calculations. A $P<.05$ was considered statistically significant. We used a receiver operating characteristic curve to evaluate the diagnostic capability of the ADC value for differentiation of residual or recurrent head and neck tumor from postoperative or postradiation changes. We determined a threshold of ADC value, with which the highest accuracy was obtained for discriminating residual or recurrent head and neck tumors from postoperative or postradiation changes.

\section{Results}

The studies of 2 patients (6.2\%) showed marked susceptibility artifacts and distortion of the diffusion-weighted MR images obtained with b factor of $1000 \mathrm{~s} / \mathrm{mm}^{2}$. These 2 cases were, therefore, excluded from the study. They included 1 (12.5\%) of the 8 lesions in the region of the paranasal sinuses and nasal cavity and 1 (25\%) of the 4 lesions in the region of the larynx. Adequate diffusion-weighted MR images were obtained in the other 30 patients. Table 1 outlines the location and method of treatment of these 30 patients. The final diagnosis in the 30 patients who qualified for the study was residual or recurrent tumor in 19 patients and posttreatment changes in 11 patients.

Using routine MR imaging (T1WI, T2WI, and postcon- 


\begin{tabular}{lcr}
\hline \multicolumn{2}{l}{ Table 2: ADC values in residual or recurrent tumors and posttreatment changes } \\
\hline Variable & Range of ADC Value & Mean ADC Value \\
\hline Residual or recurrent tumor & $(0.83-1.65) \times 10^{-3} \mathrm{~mm}^{2} / \mathrm{s}$ & $1.17 \pm 0.33 \times 10^{-3} \mathrm{~mm}^{2} / \mathrm{s}$ \\
Posttreatment changes & $(1.10-2.44) \times 10^{-3} \mathrm{~mm}^{2} / \mathrm{s}$ & $2.07 \pm 0.25 \times 10^{-3} \mathrm{~mm}^{2} / \mathrm{s}$ \\
\hline
\end{tabular}

Note:-ADC indicates apparent diffusion coefficient.
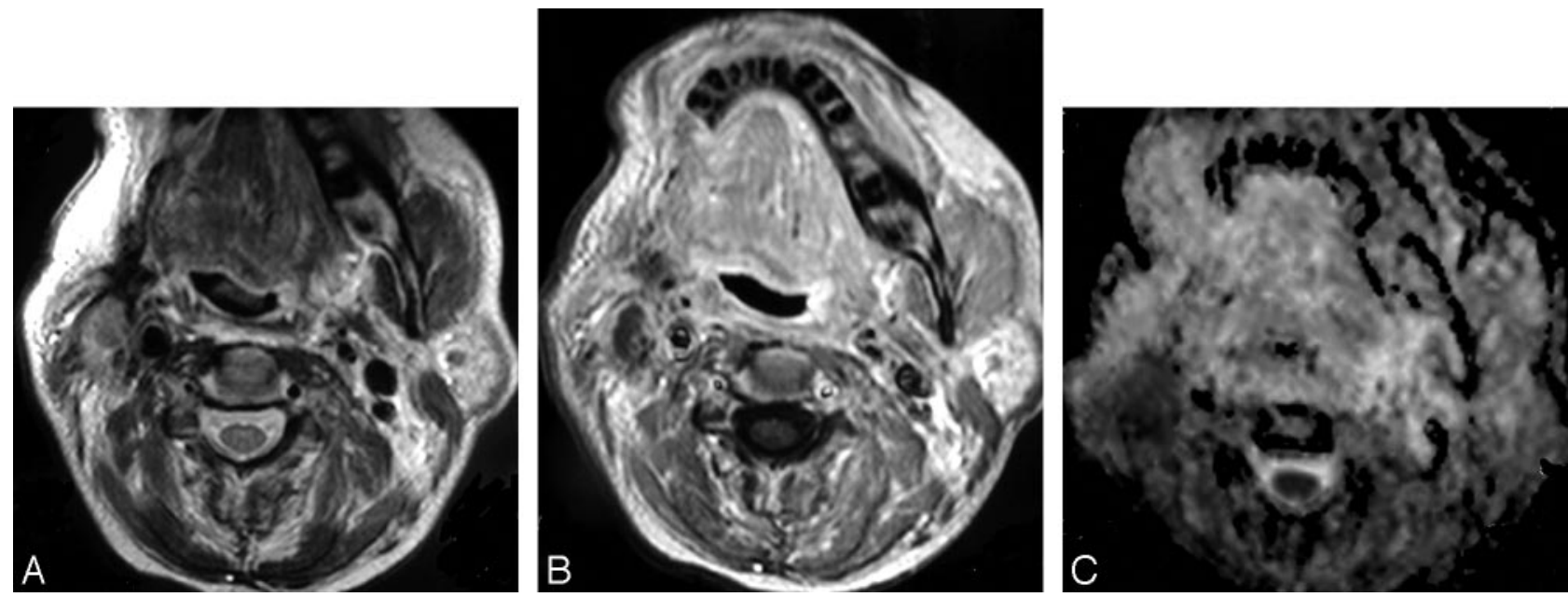

Fig 2. Recurrent oncocytic carcinoma of the right parotid gland.

$A$, Axial T2WI, shown as ill defined, is seen at the site of the right parotid region after surgical resection and irradiation.

$B$, Axial postcontrast T1WI shows the inhomogenous pattern of enhancement. Recurrence could not be excluded.

C, ADC map shows low signal intensity at the site of the lesion with a mean $A D C$ value of $1.07 \pm 0.18 \times 10^{-3} \mathrm{~mm}^{2} / \mathrm{s}$. Biopsy revealed recurrent tumor
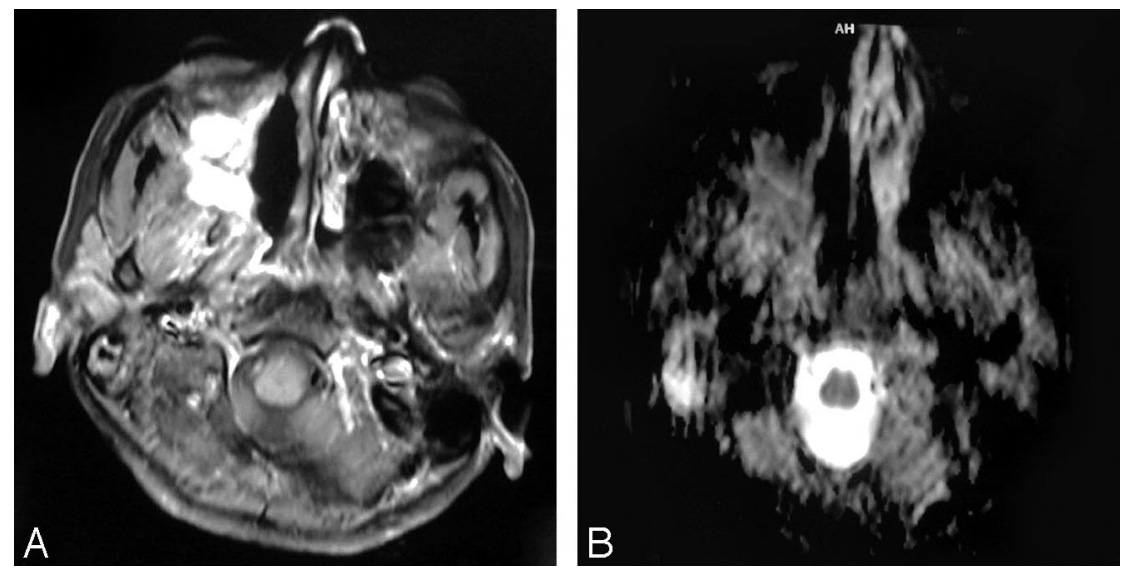

Fig 3. Recurrent squamous cell carcinoma of the nasal cavity. A, Axial postcontrast T1-weighted MR image shows that enhancing lesion is seen in the right side of the nasal cavity. Recurrent tumor cannot be differentiated from postradiation changes

$B, A D C$ map shows hypointensity within the lesion with a low $A D C$ value $\left(1.17 \pm 0.17 \times 10^{-3} \mathrm{~mm}^{2} / \mathrm{s}\right)$

trast T1WI), it was possible to diagnose tumor residual or recurrence or posttreatment changes in 16 patients $(53.3 \%)$. In the remaining 14 patients $(46.7 \%)$, a confident diagnosis of either tumor recurrence or posttreatment changes was impossible, and the final diagnosis was equivocal.

The mean ADC values recorded in the 19 patients with residual or recurrent lesions ranged from $0.83 \times 10^{-3} \mathrm{~mm}^{2} / \mathrm{s}$ to $1.65 \times 10^{-3} \mathrm{~mm}^{2} / \mathrm{s}$ with a mean value of $1.17 \pm 0.33 \times 10^{-3}$ $\mathrm{mm}^{2} / \mathrm{s}$ (Table 2). These lesions, with the exception of 2 lesions, appeared as areas of low signal intensity on the ADC map (Figs $2-4)$. Two residual lesions exhibited relatively high signal intensity on the corresponding ADC map. The smallest size of recurrent tumor detected in this study was $1.5 \mathrm{~cm}$.

Two patients with recurrent squamous cell carcinoma of the nasopharynx and oropharynx showed enlarged ipsilateral cervical lymph nodes, which showed a low ADC value close to that of the primary lesion (Fig 4). The mean ADC value of metastatic lymph node was $1.05 \pm 0.20 \times 10^{-3} \mathrm{~mm}^{2} / \mathrm{s}$. In another patient with recurrent adenoid cystic carcinoma of the maxillary sinus, diffusion-weighted MR imaging detected bone marrow infiltration as an expanded greater wing of sphenoid with area of low signal intensity and low ADC value $\left(0.84 \pm 0.30 \times 10^{-3} \mathrm{~mm}^{2} / \mathrm{s}\right)$ on the ADC map (Fig 5).

The mean ADC values noted in the 11 patients with posttreatment changes ranged from $1.10 \times 10^{-3} \mathrm{~mm}^{2} / \mathrm{s}$ to $2.44 \times$ $10^{-3} \mathrm{~mm}^{2} / \mathrm{s}$ with a mean value of $2.07 \pm 0.25 \times 10^{-3} \mathrm{~mm}^{2} / \mathrm{s}$ (Table 2). Posttherapeutic soft tissue changes appeared, except in 3 cases, as areas of high signal intensity on the ADC map and showed significant loss of their signal intensity on the diffusion-weighted MR images obtained at b factor of $1000 \mathrm{~s} / \mathrm{mm}^{2}$ (Fig 6). In 3 patients, postradiation changes showed only mild loss of signal intensity on the diffusion-weighted MR images obtained with $\mathrm{b}$ factor of $1000 \mathrm{~s} / \mathrm{mm}^{2}$ and appeared as areas of low/mixed signal intensity on the ADC map (Fig 7). 

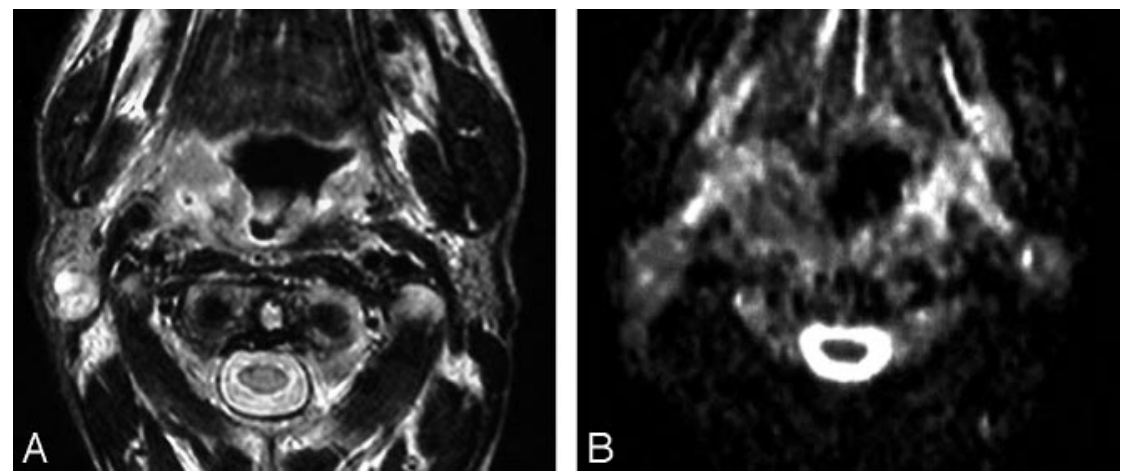

Fig 4. Recurrent squamous cell carcinoma of the oropharynx with metastatic cervical lymph node.

A, Axial T2WI shows an ill-defined irregular mass of inhomogeneous signal intensity involving the right side of the oropharynx. An enlarged cervical lymph node (arrow) with inhomogeneous high signal intensity is also noted at the right side of the neck.

$B, A D C$ map shows low signal intensity of both the lesion and the lymph node with a mean ADC value of $1.20 \pm 0.22 \times$ $10^{-3} \mathrm{~mm}^{2} / \mathrm{s}$ and $1.05 \pm 0.20 \times 10^{-3} \mathrm{~mm}^{2} / \mathrm{s}$, respectively, suggestive of tumor recurrence with metastatic lymph nodes. This was proved by biopsy.
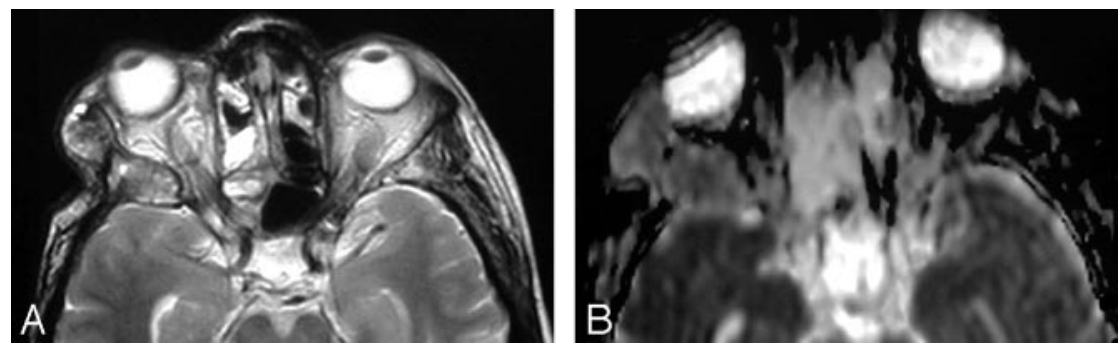

Fig 5. Bone marrow infiltration

A, Axial T2-weighted MR image shows an ill-defined inhomogeneous signal intensity involving the right greater wing of the sphenoid bone.

$B, A D C$ map shows low signal intensity at this region with a mean $A D C$ value of $0.84 \pm 0.30 \times 10^{-3} \mathrm{~mm}^{2} / \mathrm{s}$, suggestive of tumor infiltration, which was proved on bone marrow biopsy.
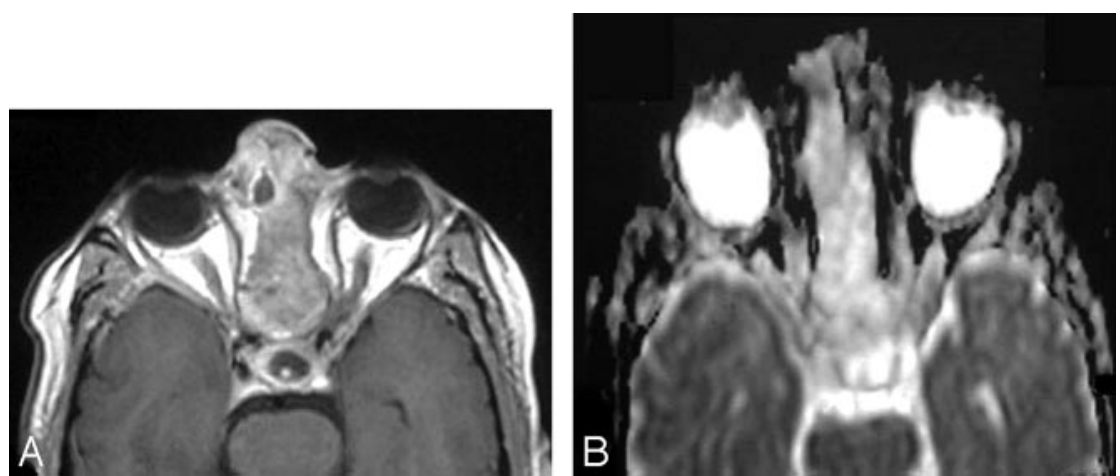

Fig 6. Posttreatment changes after surgery and radiation therapy.

A, Axial postcontrast T1WI shows an ill-defined, mildly enhancing mass at the region of the ethmoidal sinuses. Recurrence was suspected.

$B, A D C$ map shows high signal intensity at the site of the lesion denoting posttreatment fibrous tissue, which was proved by biopsy. The mean ADC value at the site of the posttreatment changes was $1.89 \pm 0.19 \times 10^{-3} \mathrm{~mm}^{2} / \mathrm{s}$.
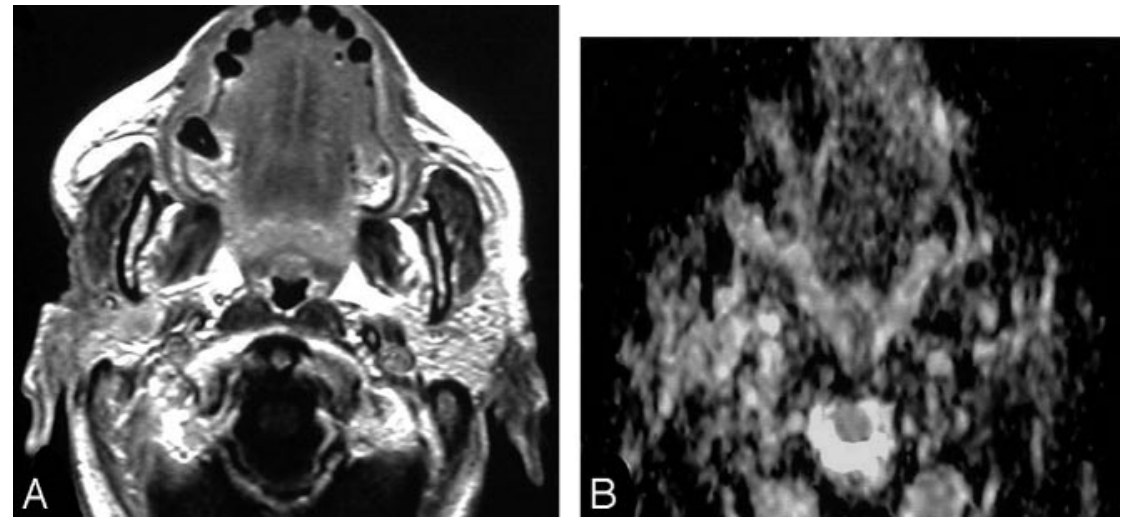

Fig 7. Posttreatment changes after surgery and radiation therapy.

A, Axial postcontrast T1WI shows a small ill-defined enhancing lesion at the site of the right parotid gland after surgical resection and irradiation. Recurrence could not be differentiated from posttreatment changes.

$B, A D C$ map shows low signal intensity at the site of the lesion with a mean $A D C$ value of $1.07 \pm 0.18 \times 10^{-3}$ $\mathrm{mm}^{2} / \mathrm{s}$. Biopsy revealed only attenuated fibrous tissue with no tumor cells.

The difference between ADC values of recurrent tumor and postoperative or postradiation changes was statistically significant $(P<.001)$. Figure 8 shows a box and whisker plot of the mean ADC values for both groups of pathology. Despite the statistical difference between the mean values of both groups, the plot reveals a relatively broad range of overlapping values in $\mathrm{ADC}$ measurements. When an $\mathrm{ADC}$ value of $1.30 \times$ $10^{-3} \mathrm{~mm}^{2} / \mathrm{s}$ was used for differentiating tumor recurrence from treatment-induced changes, the highest accuracy of $87 \%$ was obtained, with $84 \%$ sensitivity, $90 \%$ specificity, $94 \%$ pos- itive predictive value, and $76 \%$ negative predictive value (Table 3).

\section{Discussion}

Treatment options for malignant tumors of the head and neck include surgery and radiation therapy, alone or in combination. Both treatment modalities can produce edema, fibrousinflammatory reaction, and scarring of the adjacent normal soft tissues. ${ }^{2,15}$ On routine MR imaging, residual or recurrent lesions and treatment-induced changes show similar imaging 


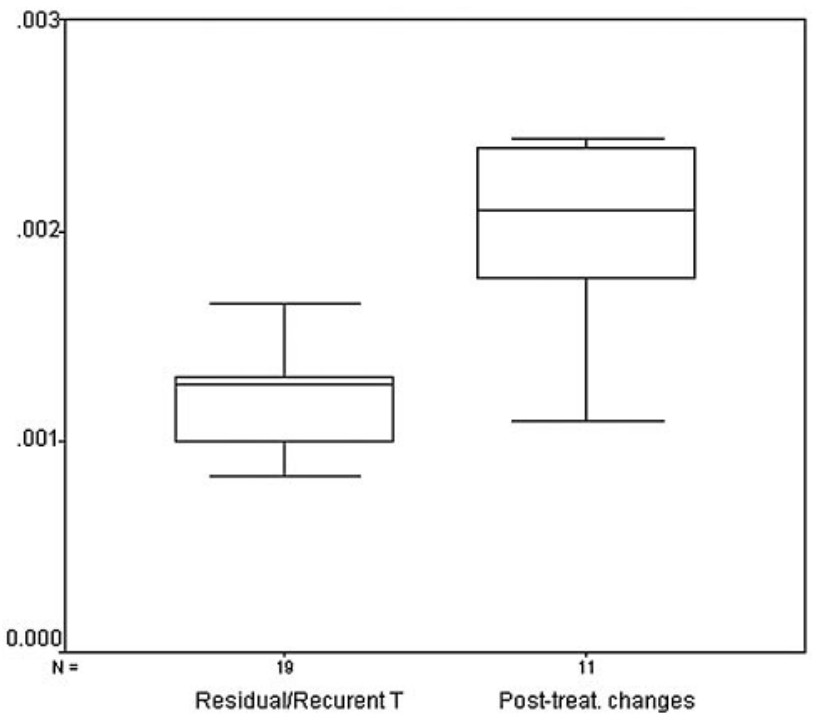

Fig 8. Box and whisker plot compares the mean ADCs of residual or recurrent tumors and posttreatment changes. The horizontal line is the median (50th percentile) of the measured values, the top and bottom of the box represent the 25th and 75th percentiles, respectively, and whiskers indicate the range from the largest to smallest observed data points. Note that despite the overlap between the ADC values of both groups, the ADCs of posttreatment changes are significantly higher than that of residual or recurrent tumors.

characteristics and are, therefore, difficult to distinguish. These characteristics include proximity to the tumor site; areas of T2 prolongation that may represent variable degrees of edema, fibrous-inflammatory reaction, or neoplasm; enhancement after the administration of gadolinium-diethylene-triaminepentaacetic acid; and variable degrees of mass effect. Areas of abnormal enhancement are considered particularly worrisome for residual or recurrent tumors. Some residual or recurrent lesions do not enhance after contrast administration, making them indistinguishable from other posttreatment changes. ${ }^{12,15,16}$

Diffusion-weighted MR imaging uses strong magnetic field gradients to make the MR imaging signal sensitive to the molecular motion of water. The information provided reflects the viability and structure of tissue on a cellular level. The ability of diffusion-weighted MR imaging to probe the intracellular milieu led to the current strong belief that diffusion-weighted MR imaging and ADC measurement could distinguish neoplastic from nonneoplastic tissue necrosis. ${ }^{11}$ Baur et al ${ }^{11}$ evaluated the value of diffusion-weighted MR imaging in the differentiation of recurrent soft tissue tumors of the musculoskeletal system from radiation-induced changes. Herneth et $\mathrm{al}^{17}$ used ADC measurement for the same purpose in a squamous cell carcinoma tumor model in animals. Hein et $\mathrm{al}^{12}$ found a statistically significant difference between the ADC value of recurrent brain tumors and treatment-induced changes. The present study, to the best of our knowledge, is the first report on the value of diffusion-weighted MR imaging for differentiating residual or recurrent head and neck tumors from posttreatment changes.

Diffusion-weighted echo-planar pulse sequence is the sequence of choice for the quantitative study of diffusion, because the diffusion and relaxation effects contribute separately to the MR signal intensity and can be easily separated. ${ }^{18}$ Furthermore, EPI is a very fast technique that enables data acqui- sition, with different b-values, within a reasonably short time. The main trade-off of the EPI pulse sequence is that it is very sensitive to magnetic susceptibility effects, resulting in geometric distortion artifacts that tend to be more severe with increasing b values. ${ }^{6,18,19}$ Two (6.2\%) of our studies showed marked distortion and susceptibility artifacts and were, therefore, excluded from the final analysis. They included $12.5 \%$ of the studies at the paranasal sinus region and $25 \%$ of the studies at the region of the larynx. Wang et $\mathrm{al}^{7}{ }^{7}$ using a diffusionweighted echo-planar pulse sequence similar to that used in our study, reported marked susceptibility artifacts in $42 \%$ of their studies in the region of the paranasal sinuses and $23 \%$ of their studies in the region of the larynx. Yoshino et $\mathrm{al}^{13} \mathrm{re}-$ ported that diffusion-weighted echo-planar MR imaging of the head and neck are liable to severe susceptibility artifacts because of the numerous air spaces within the head and neck, including sinonasal spaces, mastoid air cells, and aerodigestive tracts.

Our data demonstrated that the mean ADC values for residual or recurrent tumors were significantly lower $(P<.001)$ than that for posttreatment changes. Residual or recurrent lesions appeared as areas of low signal intensity on the ADC map. On the other hand, posttreatment changes appeared as areas of high signal intensity on the ADC map. Using diffusion-weighted MR imaging, Baur et al $^{11}$ found a statistically significant difference in the degree of signal intensity reduction exhibited by posttreatment changes (more signal loss) as compared with residual or recurrent malignant tumors (less signal loss) of the musculoskeletal system. The differences in ADC values reflect the distinct difference in the histopathologic features and water proton distribution of tumors and posttreatment soft tissue changes. Malignant tumors have enlarged nuclei, hyperchromatism, and show hypercellularity. These histologic characteristics reduce the diffusion space of water protons in both the extracellular and intracellular dimensions with a resultant decrease in the ADCs. ${ }^{7,12}$ On the other hand, tissues with posttreatment changes show relatively low cellularity associated with variable degrees of edema and inflammatory reaction that are characterized by an increase of the interstitial water content, where less barriers for diffusion exist, with a subsequent increase in their ADCs. ${ }^{7,11}$

The results in this study showed a zone of overlap between the ADC values of the residual or recurrent lesions and posttreatment changes. If an ADC value of $1.3 \times 10^{-3} \mathrm{~mm}^{2} / \mathrm{s}$ was chosen as a threshold value to differentiate between residual lesions and posttreatment changes, the best results would have been achieved with an overall accuracy of $87 \%$, sensitivity of $84 \%$, specificity of $90 \%$, positive predictive value of $94 \%$, and negative predictive value of $76 \%$. Similarly, Hein et $\mathrm{al}^{12}$ found a wide range of overlap between the mean ADC values for recurrent tumors and postradiation changes in the brain. Wang et $\mathrm{al}^{7}$ reported that a threshold ADC value of $1.22 \times$ $10^{-3} \mathrm{~mm}^{2} / \mathrm{s}$ for differentiating benign and malignant tumors of the head and neck has $87 \%$ accuracy, $84 \%$ sensitivity, $91 \%$ specificity, 93\% positive predictive value, and $78 \%$ negative predictive value. Abdel Razek et $\mathrm{al}^{20}$ reported that the mean $\mathrm{ADC}$ value of the viable and necrotic part of the head and neck tumor are $1.17 \pm 0.33 \times 10^{-3} \mathrm{~mm}^{2} / \mathrm{s}$ and $2.11 \pm 0.58 \times 10^{-3}$ $\mathrm{mm}^{2} / \mathrm{s}$, respectively.

False-negative and false-positive results were seen in 5 


\begin{tabular}{|c|c|c|c|c|c|}
\hline $\begin{array}{l}\text { Threshold of ADC Value } \\
\left(\times 10^{-3} \mathrm{~mm}^{2} / \mathrm{s}\right)\end{array}$ & $\begin{array}{l}\text { Sensitivity } \\
\%(n / M)\end{array}$ & $\begin{array}{l}\text { Specificity } \\
\%(n / M)\end{array}$ & $\begin{array}{c}\text { Accuracy } \\
\%\end{array}$ & $\begin{array}{l}\text { Positive Predictive } \\
\text { Value } \%(n / M)\end{array}$ & $\begin{array}{l}\text { Negative Predictive } \\
\text { Value } \%(n / M)\end{array}$ \\
\hline$\leq 1.00$ & $37(7 / 19)$ & $100(11 / 11)$ & 60 & $100(7 / 7)$ & $47(11 / 23)$ \\
\hline$\leq 1.30$ & $84(16 / 19)$ & $90(10 / 11)$ & 87 & $94(16 / 17)$ & $76(10 / 13)$ \\
\hline$\leq 1.50$ & $89(16 / 19)$ & $73(9 / 11)$ & 83 & $85(17 / 20)$ & $80(8 / 10)$ \\
\hline$\leq 2.00$ & $100(19 / 19)$ & $45(5 / 11)$ & 80 & $76(19 / 25)$ & $100(5 / 5)$ \\
\hline$\leq 2.40$ & $100(19 / 19)$ & $9(1 / 11)$ & 66 & $65(19 / 29)$ & $100(1 / 1)$ \\
\hline
\end{tabular}

Note:-ADC indicates apparent diffusion coefficient.

(17\%) of our cases. All 5 of the cases showed mixed or intermediate (mildly low to mildly high) signal intensity on the corresponding ADC map. Two of these cases were residual lesions that showed a relatively high $\mathrm{ADC}$ value (range $=$ $[1.40-1.65] \times 10^{-3} \mathrm{~mm}^{2} / \mathrm{s}$ ) and were falsely diagnosed as posttreatment changes. The mean ADC value obtained for nontreated head and neck cancers in other studies ${ }^{7}$ was slightly lower than that in our study $\left(1.13 \times 10^{-3} \mathrm{~mm}^{2} / \mathrm{s}\right)$. The relatively high ADC values for residual lesions in our study could be due to the edema and small areas of liquefactive necrosis induced by radiation. Matzek et $\mathrm{al}^{21}$ reported a statistically significant increase in the ADC value of squamous cell carcinoma of the oropharynx 10 days after radiation therapy $\left(0.71 \pm 0.19 \mathrm{~mm} / \mathrm{s}^{2}\right.$ before treatment versus $2.05 \pm 0.43$ $\mathrm{mm} / \mathrm{s}^{2}$ after treatment). They attributed this increase to areas of micronecrosis and edema developing within the tumor. Another contributing factor for the increased ADC in our study could be the diverse pathologies in our study compared with the more homogenous pathology in the work of Wang et al. ${ }^{7}$

The remaining 3 cases were posttreatment changes that showed a relatively low ADC value (range $=[1.10-1.60] \times$ $10^{-3} \mathrm{~mm}^{2} / \mathrm{s}$ ) and were falsely mistaken for tumor residual or recurrence. Retrospective analysis revealed that these patients received radiation therapy more than a year before. Nomayr et $\mathrm{al}^{15}$ reported that during the first 6 months after radiation therapy, the dominant postradiation changes include edema, active fibrous-inflammatory reaction, and increased vascular permeability due to endothelial damage. Later (6-24 months after radiation), these changes will show gradual regression, and the dominant reaction will be attenuated fibrous reaction with restriction of water diffusion. Hein et $\mathrm{al}^{12}$ added that the different contributions of different components, such as necrosis, fibrous scar tissue, and granulation tissue to the ADC after therapy, have not been determined thus far in absolute values. This could explain the great variability of ADCs found among our patients with posttreatment changes and account for the relatively low ADC values found in 4 of these patients.

The proper choice of areas of sampling for calculation of $\mathrm{ADC}$ values is a very important factor to reduce false results. Precautions during ADC measurement include the following: all areas of gross necrosis should be excluded from the sample (ROI), a freehand-drawn ROI should be used if possible, and, finally, multiple ROIs with a mean value should be tried if the lesion shows significant signal intensity heterogeneity. However, volume averaging and areas of micronecrosis cannot be avoided during ADC measurements and will continue to be a source of sampling error.

Recurrent disease can be within ipsilateral or contralateral nodes. ${ }^{22}$ Two of our patients with recurrent squamous cell carcinoma of the nasopharynx and oropharynx showed en- larged ipsilateral cervical lymph nodes. The enlarged lymph nodes showed a low ADC value $\left(1.05 \pm 0.20 \times 10^{-3} \mathrm{~mm}^{2} / \mathrm{s}\right)$, close to that of the primary lesion $\left(1.20 \pm 0.22 \times 10^{-3} \mathrm{~mm}^{2} /\right.$ s), denoting the metastatic nature of these nodes. It is plausible that the cellularity of metastatic cancer in the nodes might not differ greatly from that of the primary lesion, which is the case in our study. Sumi et $\mathrm{al}^{14}$ showed that ADC calculated on diffusion-weighted MR images successfully discriminated metastatic lymph nodes in the neck from benign reactive lymphadenopathy. The ADC value calculated for metastatic lymph nodes in the work of Sumi et $\mathrm{al}^{14}\left(0.410 \pm 0.105 \times 10^{-3}\right.$ $\mathrm{mm}^{2} / \mathrm{s}$ ) was much lower than that in our study. However, this could be due to the difference in the diffusion factor, factor $b$, used in both studies.

Bone marrow displays variable patterns of signal intensity depending on the proportion of fat cells and cellular marrow present. Signal intensity characteristics of hematopoietic marrow on different pulse sequences are not specific and may be difficult to distinguish from those of neoplastic or other diseases. So, biopsy is often necessary to establish a definite diagnosis. ${ }^{10}$ In this study, diffusion-weighted images delineated the extent of bone marrow infiltration of the skull base and greater wing of sphenoid in 1 patient with recurrent adenoid cystic carcinoma of the maxillary sinus much better than the routine MR images. Tumor infiltration appeared as areas of low signal intensity on the ADC map with a low ADC value.

One important limitation in this study was the heterogeneity of the patient population. A large number of lesions with different locations, pathologic conditions, type of treatment, and duration between imaging and treatment were included in our study to obtain data that could be analyzed statistically; however, this was at the expense of the homogeneity of our results, which makes generalization difficult. However, we think that the location of the lesion might not have had significant impact on the ADC value. Although standardization of duration after treatment might have a theoretic impact on results, it is difficult and does not appear to be practical. Difference in pathology and type of treatment would definitely affect the ADC value, because tumors with high cellularity are expected to have low ADC values, whereas those with low cellularity are expected to have high ADC values. On the other hand, postsurgical changes with edema and/or fibrosis would have a different ADC value than postradiation changes with edema and/or necrosis. Further studies that include more homogenous study samples are required to further address the impact of these variables on the ADC value.

\section{Conclusions}

Diffusion-weighted MR imaging with ADC measurement has promising results for differentiating residual or recurrent head 
and neck tumors from postoperative or postradiation changes. It has better results than conventional MR imaging. ADC value can be used a guide for tissue sampling. The technique has very short scanning time and adds no extra cost to patients who are already undergoing MR imaging. It can be used for follow-up of these patients after treatment.

\section{References}

1. Castelijns J, van den Brekel M, Tobi H, et al. Laryngeal carcinoma after radiation therapy: correlation of abnormal MR imaging signal patterns in laryngeal cartilage with the risk of recurrence. Radiology 1996;198:151-55

2. Farber L, Benard F, Machtay M, et al. Detection of recurrent head and neck squamous cell carcinoma after radiation therapy with $2-{ }^{18} \mathrm{~F}$-fluoro-2-deoxyD-glucose positron emission tomography. Laryngoscope 1999;109:970-75

3. Hermans R, Pameijer F, Mancouso A, et al. Laryngeal or hypopharyngeal squamous cell carcinoma: can follow-up CT after definitive radiation therapy be used to detect local failure earlier than clinical examination alone. Radiology 2000;214:683-87

4. Lai P, Yang C, Pan H, et al. Recurrent inverted papilloma: diagnosis with pharmacokinetic dynamic gadolinium-enhanced MR imaging. AJNR Am J Neuroradiol 1999;20:1445-51

5. Chisin R, Macapinlac H. The indications of FDG-PET in neck oncology. Radiol Clin North Am 2000;38:999-1012

6. Schaefer P, Grant P, Gonzalez R. Diffusion-weighted MR imaging of the brain. Radiology 2000;217:331-45

7. Wang J, Takashima S, Takayama F, et al. Head and neck lesions: characterization with diffusion-weighted echo-planar MR imaging. Radiology 2001;220:621-30

8. Lang $\mathrm{P}$, Wendland $\mathrm{M}$, Saeed $\mathrm{M}$, et al. Osteogenic sarcoma: noninvasive in vivo assessment of tumor necrosis with diffusion-weighted MR imaging. Radiology 1998;206:227-35

9. Kim T, Murakami T, Takahashi S, et al. Diffusion-weighted single-shot echoplanar MR imaging for liver disease. AJR Am J Roentgenol 1999;173:393-98

10. Maeda M, Sakuma H, Maier S, et al. Quantitative assessment of diffusion abnormalities in benign and malignant vertebral compression fractures by line scan diffusion-weighted imaging. AJR Am J Roentgenol 2003;181:1203-09
11. Baur A, Huber A, Arbogast S, et al. Diffusion-weighted imaging of tumor recurrences and posttheraputical soft tissue changes in humans. Eur Radiol 2001;11:828-33

12. Hein P, Eskey C, Dunn J, et al. Diffusion-weighted imaging in the follow-up of treated high-grade gliomas: tumor recurrence versus radiation injury. AJNR Am J Neuroradiol 2004;25:201-09

13. Yoshino N, Yamada I, Ohbayashi N, et al. Salivary glands and lesions: evaluation of apparent diffusion coefficient with split echo diffusion weighted MR imaging -initial results. Radiology 2001;221:837-42

14. Sumi M, Sakihama N, Sumi T, et al. Discrimination of metastatic lymph nodes with diffusion-weighted MR imaging in patients with head and neck cancer. AJNR Am J Neuroradiol 2003;24:1627-34

15. Nomayr A, Lell M, Sweeney R, et al. MRI appearance of radiation-induced changes of normal cervical tissues. Eur Radiol 2001;11:1807-17

16. Baba $Y$, Yamashita $Y$, Onomichi M, et al. Dynamic magnetic resonance imaging of head and neck lesions. Top Magn Reson Imaging 1999;10:125-29

17. Herneth A, Bammer R, Bednarski M, et al. The role of line-scan-diffusionimaging in the characterization of viable tumor and scar tissue: correlation of quantitative diffusion measurements with histopathologic and genomics data. Paper presented at: Scientific Assembly and Annual Meeting of Radiological Society of North America, 2002; Chicago, Ill.

18. Le Bihan DJ. Differentiation of benign versus pathologic compression fractures with diffusion-weighted MR imaging: a closer step toward the "holy grail" of tissue characterization. Radiology 1998;207:305-07

19. Rowley H, Grant E, Roberts T. Diffusion MR imaging. Theory and application. Neuroimaging Clin North Am 1999;9:343-61

20. Abdel Razek A, Tawfik A, Rizk N. Role of diffusion trace MR imaging in differentiation between viable and necrotic part of head and neck tumors. Paper presented at: Scientific Assembly and Annual Meeting of Radiological Society of North America, 2002; Chicago, Ill.

21. Matzek W, Czerny C, Krestan C, et al. Non-invasive therapy monitoring with diffusion weighted imaging: evaluation of effect of radiochemotherapy in squamous cell carcinoma of the oropharynx. Paper presented at: XVI International Congress of Head \& Neck Radiology, September 2003; Frankfurt/Main, Germany.

22. Wester $D$, Whiteman $M$, Singer $S$, et al. Imaging of the post-operative neck with emphasis on the surgical flaps and their complications. AJR Am J Roentgenol 1995;164:989-93 